\title{
ON THE SIMULTANEOUS SOLUTION OF A CERTAIN SYSTEM OF LINEAR INEQUALITIES
}

GEORGE J. MINTY

The author recently proved the following theorem:

THEOREM 1. Let $X$ be a Hilbert space, with real or complex scalars and inner product $\langle x, y\rangle$. Let $x_{1}, \cdots, x_{m}$ and $y_{1}, \cdots, y_{m}$ be given such that

$$
\operatorname{Re}\left\langle x_{i}-x_{j}, y_{i}-y_{j}\right\rangle \geqq 0 \quad(i, j=1, \cdots, m),
$$

and let $x$ be any point of $X$. Then there exists $a$ point $y$ such that

$$
\operatorname{Re}\left\langle x_{i}-x, y_{i}-y\right\rangle \geqq 0 \quad(i=1, \cdots, m) .
$$

The proof was patterned after Schoenberg's [3] proof of Kirszbraun's theorem. B. Grünbaum [2] has generalized my and Schoenberg's proofs to obtain a theorem which incorporates Theorem 1 and Kirszbraun's theorem, and J. G. Wendel has contributed a neater proof of Theorem 1. With Professor Wendel's permission, I reproduce his proof:

Lemma. Let $\mathfrak{X}$ be $E^{n}$, with the usual (real) scalars and inner product, and let $x_{1}, \cdots, x_{m} ; y_{1}, \cdots, y_{m}$ be given such that

$$
\left\langle x_{i}-x_{j}, y_{i}-y_{j}\right\rangle \geqq 0 \quad(i, j=1, \cdots, m) .
$$

Then there exists a point $y$ such that

$$
\left\langle x_{i}, y\right\rangle \leqq\left\langle x_{i}, y_{i}\right\rangle \quad(i=1, \cdots, m) .
$$

Proof of The lemma. Let $A$ be the matrix whose $i$ th row is $x_{i}$, and let $b$ be the column-vector whose $i$ th element is $\left\langle x_{i}, y_{i}\right\rangle$. Then $\left(2^{\prime}\right)$ is equivalent to $A y \leqq b$. If there is no solution for $y$, then by Stiemke's Theorem [1, Theorem 2.7] there exists a row-vector $\eta \leqq 0$ such that

$$
\eta A=0 \text { and } \eta b=1 .
$$

Suppose this to be the case. Then (3a) implies $\sum_{i} \eta_{i} x_{i}=0$, hence for each $j$,

$$
\sum_{i} \eta_{i}\left\langle x_{i}, y_{j}\right\rangle=0
$$

Also (3b) implies

Received by the editors January 11, 1961. 


$$
\sum_{i} \eta_{i}\left\langle x_{i}, y_{i}\right\rangle=1
$$

Expanding ( $\left.1^{\prime}\right)$, multiplying by $\eta_{i} \eta_{j}$ and summing on $i, j$, we have

$$
\begin{aligned}
\sum_{i} \eta_{i}\left\langle x_{i}, y_{i}\right\rangle \sum_{j} \eta_{j}+\sum_{i} \eta_{i} \sum_{j} \eta_{j}\left\langle x_{j}, y_{j}\right\rangle \\
\geqq \sum_{j} \eta_{j} \sum_{i} \eta_{i}\left\langle x_{i}, y_{j}\right\rangle+\sum_{i} \eta_{i} \sum_{j} \eta_{j}\left\langle x_{j}, y_{i}\right\rangle
\end{aligned}
$$

i.e., by (4) and (5), $2 \sum_{j} \eta_{j} \geqq 0$. But $\eta \leqq 0$ and some $\eta<0$ by (3b). We have a contradiction, and there is at least one solution for $y$.

Proof of Theorem 1. First, we take up the case where $\mathfrak{X}=E^{n}$, and set $x_{i}^{\prime}=x_{i}-x$; the conclusion follows by application of the Lemma to $x_{1}^{\prime}, \cdots, x_{m}^{\prime}$ and $y_{1}, \cdots, y_{m}$.

We next suppose that $\mathfrak{X}$ is any finite-dimensional Hilbert space. If the scalars are complex, it is easily verified that $[x, y]=\operatorname{Re}\langle x, y\rangle$ is real inner product provided the scalar product $\alpha \cdot x$ is restricted to real $\alpha$, and that the resulting Hilbert space with real scalars is of dimension $2 n$. The conclusion now follows from the isomorphism with $E^{n}$ or $E^{2 n}$.

In case $\mathfrak{X}$ is infinite-dimensional, we simply apply the above results to the (finite-dimensional) subspace spanned by $x_{1}, \cdots, x_{m}$; $y_{1}, \cdots, y_{m}$ and $x$.

\section{REFERENCES}

1. D. Gale, The theory of linear economic models, McGraw-Hill, New York, 1960.

2. B. Grunbaum, A generalization of theorems of Kirszbraun and Minty, Proc. Amer. Math. Soc. (to appear).

3. I. J. Schoenberg, On a theorem of Kirzbraun and Valentine, Amer. Math. Monthly 60 (1953), 620-622.

UNIVERSITY OF MichigaN 